\title{
THE DWARF CEPHEIDS
}

\author{
D. H. MCNAMARA and W. R. LANGFORD \\ Dept. of Physics and Astronomy, Brigham Young University, Provo, Utah, U.S.A.
}

\begin{abstract}
Intermediate-band photometry has been used to derive effective temperatures, metal abundances, and surface gravities of nine stars of the dwarf Cepheid group of variable stars. A strong correlation exists between the metal-line strengths and the period of pulsation in the sense that the shorter-period stars have weaker metal lines. The dwarf Cepheids are evidentally found among both population I and population II stars.

The surface gravities and effective temperatures have been used in conjunction with pulsation theory to calculate masses and absolute magnitudes. The masses derived for the shorter-period stars are $\lesssim 1 M_{\odot}$ in agreement with earlier estimates, but the longer-period stars are found to have masses in the range of 2-3 $M_{\odot}$. We also find that the dwarf 'Cepheids exhibit a period-luminosity relation.

The spectra of the dwarf Cepheids indicate that all of them have small rotational velocities, $v \sin i \leqslant 20 \mathrm{~km} \mathrm{~s}^{-1}$. We have also detected the Li line $\lambda 6707$ in the spectrum of VZ Cnc.

The observational evidence indicates that the dwarf Cepheids are post main-sequence stars that have not yet entered the red-giant stage, as suggested by Eggen, rather than stars in a post-red-giant evolutionary stage as was generally believed heretofore.
\end{abstract}

\section{DISCUSSION}

Breger: Where do you think the RR Lyrae stars start and the dwarf Cepheids stop?

McNamara: I have a feeling that the strong line RR Lyrae stars are simply an extension of this group. I'm not in a position to guarantee that, but I'm going to look for lithium in my strong line RR Lyrae stars, hopefully this fall, and I wouldn't be a bit surprised to find it.

Bessell: The problem with this metallicity criterion is that amongst the short period group there are some which do have young disc space motions and amongst the long period group there are some which have old disc space motions. So if you define them as old disc on the basis of the space motions then that picture doesn't hold; I agree with the abundances, but from the space motions the picture doesn't hold together too well.

McNamara: For this particular group that I've observed, at least abundancewise, the $\Delta m_{1}$ correlation seems very good. Of the stars that I had available I think there were only 3 with high velocities, and I haven't looked into that motion problem for the longer period groups.

Sinvhal: One of us recently worked on SZ Lyncis and came out with a mass of about 1.2 solar masses as a result.

McNamara: I see. I think that could agree very well with where I'd place it.

Stobie: What is your explanation for the high metal abundance group, why is there so few dwarf Cepheids compared to $\delta$ Scuti stars?

McNamara: I think it's the rotational velocity, again this may be just a classification problem as indicated this morning. If you plot the number of stars versus the rotational velocities, you find that there's a pretty flat distribution here for the $\delta$ Scuti variables. On the other hand, the stars of the type we've been talking about here with these large amplitudes all have very small $v \sin i$. The $\delta$ Scuti variables are distributed all along here, and some of the stars that have been classified as $\delta$ Scuti variables I think really belong to 
this group. And the other $\delta$ Scuti variables, a very small fraction that we observed that have small rotational velocities, I think, are stars simply that are rotating fast but have the $\sin i$ factor (projection factor) such that they're thrown in to the small $v \sin i$ values. I think the rotational velocity plays the key role here.

Breger: I think for $\delta$ Scuti stars this is indeed the case because the big amplitude $\delta$ Scuti stars are sharp lined giants usually.

Iben: I'm still a bit confused. I would think there'd be a certain amount of overlap between the low metal and metal-rich group in these diagrams.

McNamara: I would agree with you, because if you're taking the stars off a zero-age main sequence you expect some of these of lower metal content to be mixed in with some of higher metal content. I'm rather surprised at how sharp this relationship is.

Iben: But is there a split into two pieces or is this a continuous thing?

McNamara: I think it is continuous. That's the thing that shocks me. It's as if we worked our way down to various zero-age main sequences. Would you get some strong line ones, then, of extreme short period?

Bessell: Yes, HD 199757.

McNamara: I'm happy about this, not unhappy.

Rodgers: Do you see it as a problem though that the rate of growth of $[\mathrm{Fe} / \mathrm{H}]$ is a function of time; $\delta \log t$ is not large. This just goes against every other piece of evidence we know about $\delta(\mathrm{Fe} / \mathrm{H}) / \delta t$, for the old disc right up to the Hyades.

McNamara: I think it works out. I think this is what you expect. 